\title{
Enfermedad celiaca y diabetes mellitus tipo 1: asociación y características clínicas
}

\section{Celiac disease and type 1 diabetes mellitus: association and clinical characteristics}

\author{
Autores: Ana Iris Ramírez Benítez ${ }^{1}$, María Carolina Miranda Ojeda ${ }^{2}$, Lorena Ferreira ${ }^{3}$, Mafalda Concepción \\ Palacios Lugo ${ }^{1}$, Jorge Tadeo Jiménez González ${ }^{1}$, Laura Flores ${ }^{1}$
}

Artículo recibido: 27 diciembre 2013

Artículo aceptado: 23 febrero 2014

\section{Resumen}

Introducción: la prevalencia de enfermedad celiaca (EC) en pacientes con diabetes mellitus tipo 1 (DM1) es de aproximadamente 4\%, con variaciones del 1 al 8\% según distintas publicaciones. En la mayoría de los casos son asintomáticos u oligosintomáticos. El impacto de la EC en el control metabólico aun no es bien conocido, sobre todo en lo que respecta a una mayor predisposición a eventos de hipoglucemia.

Objetivos: determinar la frecuencia de EC en adolescentes y adultos con DM1, relacionar la presencia de EC con las características clínicas, control metabólico y eventos de hipoglucemias.

Materiales y métodos: estudio de corte transversal, de 64 pacientes adolescentes y adultos con DM1, sin EC conocida, en quienes se realizó el dosaje de anticuerpo anti transglutaminasa y nivel de IgA. El diagnóstico de EC se basó en la presencia de anticuerpos anti transglutaminasa IgA junto a la confirmación por biopsia intestinal. Fue valorada la presencia de síntomas gastrointestinales y generales, eventos de hipoglucemia y el control metabólico por medio de la HbA1c. Se comparó a los DM1 diagnosticados de EC con un grupo control de DM1 sin EC, con edad y sexo similar.

Resultados: de los 64 pacientes, $50 \%$ eran mujeres. La edad promedio fue 25,1 $\pm 6,04$ años. La EC fue diagnosticada en 6 casos (9,37\% CI 95\% 3,5-19,3\%). Con respecto a las características clínicas, sólo 2 (33,3\%) presentaban los clásicos síntomas de EC. El promedio de HbA1c en los DM1con EC fue alto (10,01\%), pero sin diferencia significativa con el grupo control (9,9\%). Del grupo con DM1 y EC, $4(66,7 \%)$ refirieron episodios de hipoglucemia leve 1 vez por semana, sin diferencia respecto al grupo control (77,8\%).

Conclusión: se encontró una alta asociación de EC con DM1, la mayor parte se presentó en forma silenciosa o atípica, por lo que debe ser buscada regularmente, incluso en los adultos. No se halló relación entre EC y

\footnotetext{
${ }^{1}$ Departamento de Endocrinología y Diabetes de la Cátedra de Semiología Médica. Hospital de Clínicas de la Universidad Nacional de Asunción - Paraguay.

${ }^{2}$ Departamento de Gastroenterología. Hospital de Clínicas de la Universidad Nacional de Asunción - Paraguay.

${ }^{3}$ Instituto de Investigaciones en Ciencias de la Salud. Universidad Nacional de Asunción - Paraguay.

Autor de correspondencia

Dra. Ana Iris Ramírez Benítez

Departamento de Endocrinología y Diabetes de la Cátedra de Semiología Médica. Hospital de Clínicas de la Universidad Nacional de Asunción - Paraguay

Asunción 336 c/ Gral. Egusquiza, Ypané, Departamento Central, Paraguay. CP 2660.

Tel :+(595) 981873290

Correo electrónico: anitas279@hotmail.com
} 
mal control metabólico, ni con eventos de hipoglucemia.

Palabras claves: diabetes mellitus tipo 1, enfermedad celiaca, hiperglucemia, hipoglucemia.

\begin{abstract}
Introduction:The prevalence of coeliac disease (CD) among type 1 diabetic (T1DM) patients is around 4\% with variations from 1 to $8 \%$ according to different publications being most cases asymptomatic or oligosymptomatic. The impact of CD on metabolic control is not well known yet, especially regarding to a higher predisposition to hypoglycemic events.
\end{abstract}

Objectives: To determine the frequency of CD in adolescents and adults with T1DM, and relate the presence of CD with the clinical characteristics, metabolic control and hypoglycemic events.

Materials and methods: This was a cross-sectional observational study carried out in 64 adolescents and adults with T1DM who were between 15 to 40 years of age without known CD. The levels of antitransglutaminase antibodies and IgA were determined. The diagnosis of CD was based on the presence of antitransglutaminase IgA antibodies along with the confirmation by intestinal biopsy. The presence of gastrointestinal and general symptoms, hypoglycemic events and metabolic control by HbA1c were evaluated. T1DM patients diagnosed with CD were compared with a control group of T1DM without CD and both groups were matched by age and sex.

Results: Of the 64 T1DM patients, 32 were women and 32 men. Mean age was $25.1 \pm 6.04$ years. CD was confirmed by intestinal biopsy in 6 patients (9.37\%, CI 95\% $3.5-19.3 \%)$. In relation to the clinical characteristics, only 2 of them (33.3\%) presented the classic symptoms of CD. The mean HbA1c in T1DM with CD was high (10.01\%), but without a significant difference with the control group (9.9\%). In the group with T1DM and CD, 4 (66.7\%) reported episodes of mild hypoglycemia once a week with no difference in comparison with the control group (77.8\%).

Conclusion: A high association was found between CD and T1DM but most of the cases were silent or with atypical presentation. Therefore, these findings support the need of a regular screening of CD in T1DM patients even in adults ones. Association was not found between CD and a bad metabolic control or with hypoglycemic events.

Keywords: type 1 diabetes mellitus, coeliac disease, metabolic control, hypoglycemia

\title{
Introducción
}

La asociación de diabetes mellitus tipo 1 (DM1) y otras enfermedades autoinmunes, particularmente enfermedades tiroideas y enfermedad celíaca (EC), es conocida de hace mucho tiempo ${ }^{1,2}$ La prevalencia de EC en pacientes con DM1 es estimada en $4 \%$ aproximadamente ${ }^{3}$ con variaciones del 1 al $8 \%{ }^{4-9}$, superando en mucho la prevalencia de esta enfermedad en la población general, donde es de 0,5 a 1\%. La susceptibilidad genética parece ser el principal factor que explica esta asociación. El determinante de la misma se halla en la región genómica denominada HLA, caracterizada por la secuencia de genes localizados en el cromosoma 6pQ21. El HLA DR3-DQ2 y DR4-DQ8 son compartidos por la DM1 y la EC. ${ }^{10}$

La mayoría de los pacientes con DM1 presentan una forma silenciosa o subclínica de la EC y los clásicos síntomas de diarrea y dolor abdominal pueden no ser manifiestos. ${ }^{11}$ La presencia de deficiencia de hierro y acido 
fólico, con o sin anemia, baja estatura, bajo peso, retraso puberal y de la maduración ósea, diabetes de difícil control con episodios frecuentes de hipoglucemia, pueden ser las únicas manifestaciones de la EC. 2,3,10

Con respecto a la relación entre EC y control glucémico, el tema es controversial. Está descrito que pacientes con DM1 y EC tienden a un peor control glucemico ${ }^{1}$, pero según otros estudios parecería no haber diferencia entre celíacos y no celíacos en términos de hemoglobina glicosilada (HbA1c) y requerimiento total de insulina ${ }^{5,8,11,12}$ Se ha observado que pacientes con ambas patologías tienen mayores episodios de hipoglucemia y menores requerimientos de insulina, probablemente debido a la mala absorción. ${ }^{2,13}$ La incidencia de hipoglucemia en pacientes con EC no tratada es significativamente mayor con respecto a diabéticos no celiacos. La dieta libre gluten, iniciada luego de varios meses, disminuye los eventos de hipoglucemia. ${ }^{14}$

Basado en los datos expuestos más arriba, es de importancia la realización de una búsqueda periódica de EC, en todos los pacientes con DM1. El estudio inicial solicitado es el dosaje de anticuerpos. ${ }^{15-17}$ El anticuerpo antitransglutaminasa tisular IgA es el más utilizado debido a su sensibilidad. ${ }^{18-20}$ El dosaje de los niveles de IgA sérica es mandatorio, para descartar deficiencia selectiva de IgA, que puede ser observada en la EC. ${ }^{4}$ Para la confirmación del diagnóstico, la endoscopía digestiva alta con biopsia es fundamental, independientemente de la positividad de la serología. ${ }^{1,2}$

\section{Objetivos}

- Determinar la frecuencia de EC en pacientes con DM1

- Investigar las características clínicas de la EC en pacientes con DM1

- Investigar el efecto de la EC no tratada en el control metabólico de los diabéticos, por medio del nivel de HbA1c.

- Relacionar la presencia de EC con la frecuencia de eventos de hipoglucemias.

\section{Pacientes y métodos}

Estudio de corte transversal, observacional y descriptivo en pacientes con diagnóstico de DM1, que acudieron al consultorio de diabetes de la Cátedra de Semiología Médica del Hospital de Clínicas y del Programa Nacional de Diabetes, durante el periodo de junio a diciembre del 2011. Para el cálculo del tamaño de muestra se utilizó el programa informático Epi Info ${ }^{\odot}$. Para una prevalencia global esperada de $5 \%$ de $\mathrm{EC}^{3}$ en un tamaño poblacional de 50 diabéticos tipo 1, precisión 3\%, se estimó con IC de 95\% una cantidad de 40 pacientes a ser incluidos como mínimo.

El protocolo fue aprobado por el Comité de Ética en la Investigación de la Facultad de Ciencias Médicas de la U.N.A. y los pacientes y/o responsables firmaron las hojas de consentimiento o asentimiento informado. Se tomaron muestras de 64 pacientes, en forma consecutiva, a medida que acudían a la consulta ambulatoria o se internaban en la Cátedra. Se incluyeron varones y mujeres entre 15 y 40 años de edad, portadores de DM1 sin diagnóstico de EC, independientemente del tiempo de evolución de la diabetes y de la presencia de síntomas gastrointestinales o generales.

Se realizó una entrevista a cada paciente llenado por los autores y se tomaron muestras de sangre para la determinación de anticuerpos antitransglutaminasa IgA y niveles de IgA. Las determinaciones laboratoriales fueron realizadas en el laboratorio del Instituto de Investigaciones en Ciencias de la Salud de la U.N.A. por método de inmunoensayo enzimático (ELISA) e inmunodifusión radial respectivamente. Fueron considerados con serología positiva aquellos con nivel de anticuerpos antitransglutaminasa IgA mayor a $10 \mathrm{U} / \mathrm{mL}$. La deficiencia de IgA se definió por niveles menores a 90mg/dL. Los DM1 con serología positiva y aquellos con 
déficit de IgA, aún con anticuerpos negativos, fueron sometidos a endoscopía digestiva alta con toma de biopsias del intestino delgado, en el Dpto. de Gastroenterología del Hospital de Clínicas, U.N.A. El estudio anatomopatológico se realizó en la Cátedra de Anatomía Patológica del Hospital de Clínicas, U.N.A. El diagnóstico de EC se basó en las características encontradas, como atrofia vellositaria total o subtotal, hiperplasia de las criptas y proliferación de linfocitos intraepiteliales. Fueron considerados portadores de EC solo a los pacientes con anticuerpos positivos o deficiencia de IgA, y biopsia intestinal compatible con esta enteropatía. Otros datos, como antecedentes de otras patologías autoinmunes, control metabólico según HbA1c, peso, talla, IMC, presión arterial, síntomas gastrointestinales y generales, fueron relevados de las fichas clínicas. Eventos de hipoglucemia fueron rescatados por el interrogatorio, según síntomas, y clasificados de acuerdo a la frecuencia. Para las comparaciones se tomó de la cohorte inicial un grupo control de 9 DM1 negativos para EC, quienes fueron seleccionados por similar edad y sexo.

Los datos fueron analizados utilizando el paquete estadístico EPI INFO 3.5.1. Los resultados fueron expresados en forma de porcentajes para las variables nominales. Para las variables continuas: edad, tiempo de evolución de la diabetes, edad al diagnóstico de DM1, niveles de anticuerpo antitransglutaminasa tisular IgA, niveles de IgA, HbA1c, se calcularon las medias con desviaciones estándares. Para determinar asociaciones, entre variables cuantitativas se utilizó la prueba ta un nivel de significancia de 0,05.

\section{Resultados}

Participaron del estudio 64 pacientes con DM1, el promedio de edad fue de 25,1 6 6,04 (15 a 40 años) y la distribución por sexo fue equitativa, 32 femenino y 32 masculino. Las características generales de la población se resumen en la tabla 1 y los síntomas sugerentes de EC, recogidos por el interrogatorio dirigido, se observan en los gráficos 1 y 2 .

\section{TABLA 1}

\section{Características clínicas de diabéticos tipo 1 (n 64)}

\begin{tabular}{lr}
\hline Relación varón:mujer & $1: 1$ \\
Edad (años) & $25,1 \pm 6,04$ \\
Edad de diagnóstico de DM1(años) & $15,3 \pm 6,97(4-35)$ \\
Tiempo de DM1 (meses) & $115,1 \pm 90,6(1-372)$ \\
Otras enfermedades autoinmunes & $12,5 \%$ \\
Antec. familiar de autoinmunidad & $28,2 \%$ \\
Talla (m) & $1,64 \pm 0,09$ \\
IMC (k/m ${ }^{2}$ ) & $(1,45-1,72)$ \\
Hipoglucemia autoreferida & $34,4 \pm 3,1(16,4-36,4)$ \\
HbA1c & $9,1 \% \pm 2 \%$ \\
Deficiencia de IgA & $3,1 \%$ \\
Antitransglutaminasa IgA + & $7,8 \%$ \\
\hline
\end{tabular}




\section{GRÁFICO 1}

\section{Síntomas gastrointestinales de diabéticos tipo 1 (n 64)}

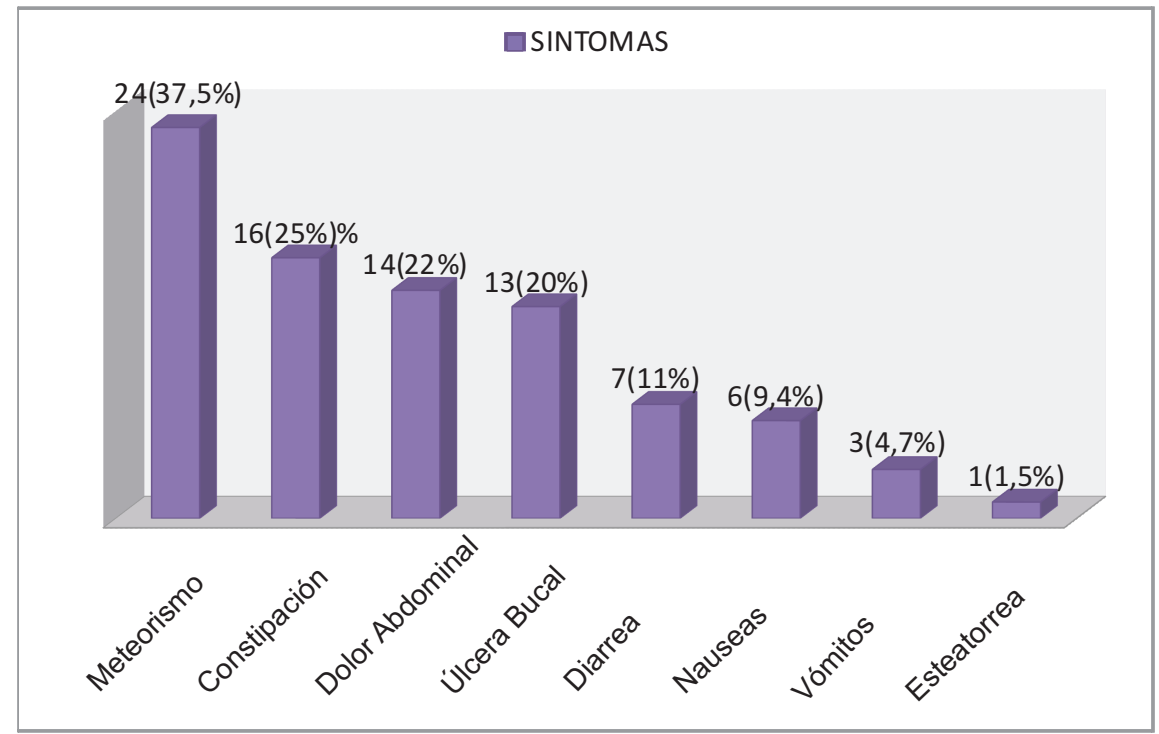

\section{GRÁFICO 2}

\section{Síntomas generales de diabéticos tipo 1 (n 64)}

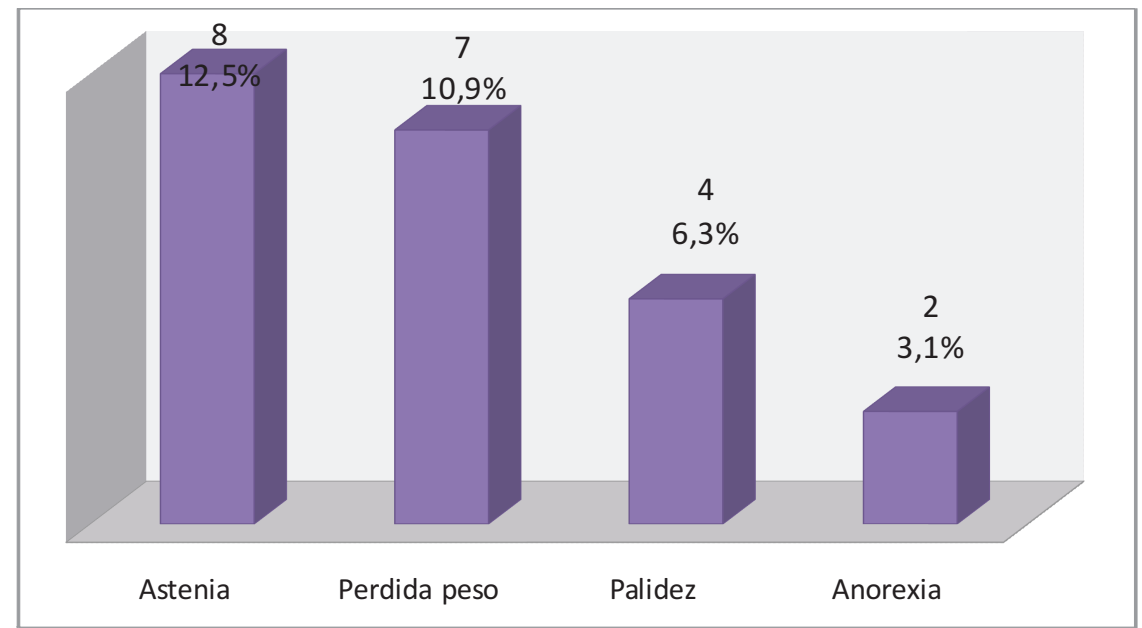

De los 64 DM1 estudiados, 5 presentaron serología positiva para EC y 2 pacientes con deficiencia de IgA. De estos 7 pacientes ( 5 mujeres y 2 varones), 6 fueron sometidos a endoscopía digestiva alta con toma de biopsia intestinal, resultando todas compatibles con EC. Una paciente no se presentó para la realización de la endoscopía (gráfico 3). La frecuencia de EC en DM1 fue de 9,37\%. 


\section{GRÁFICO 3}

\section{Población en estudio}

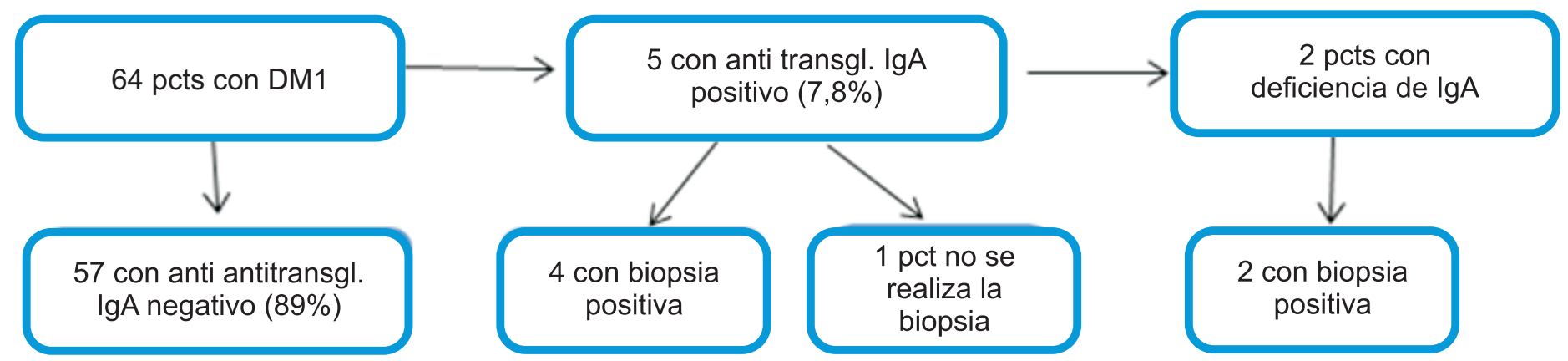

Los datos generales de pacientes con DM1 y EC se resumen en la tabla 2.

\section{TABLA 2}

\section{Características de los pacientes con DM1 y antitransglutaminasa positivo o déficit de IgA}

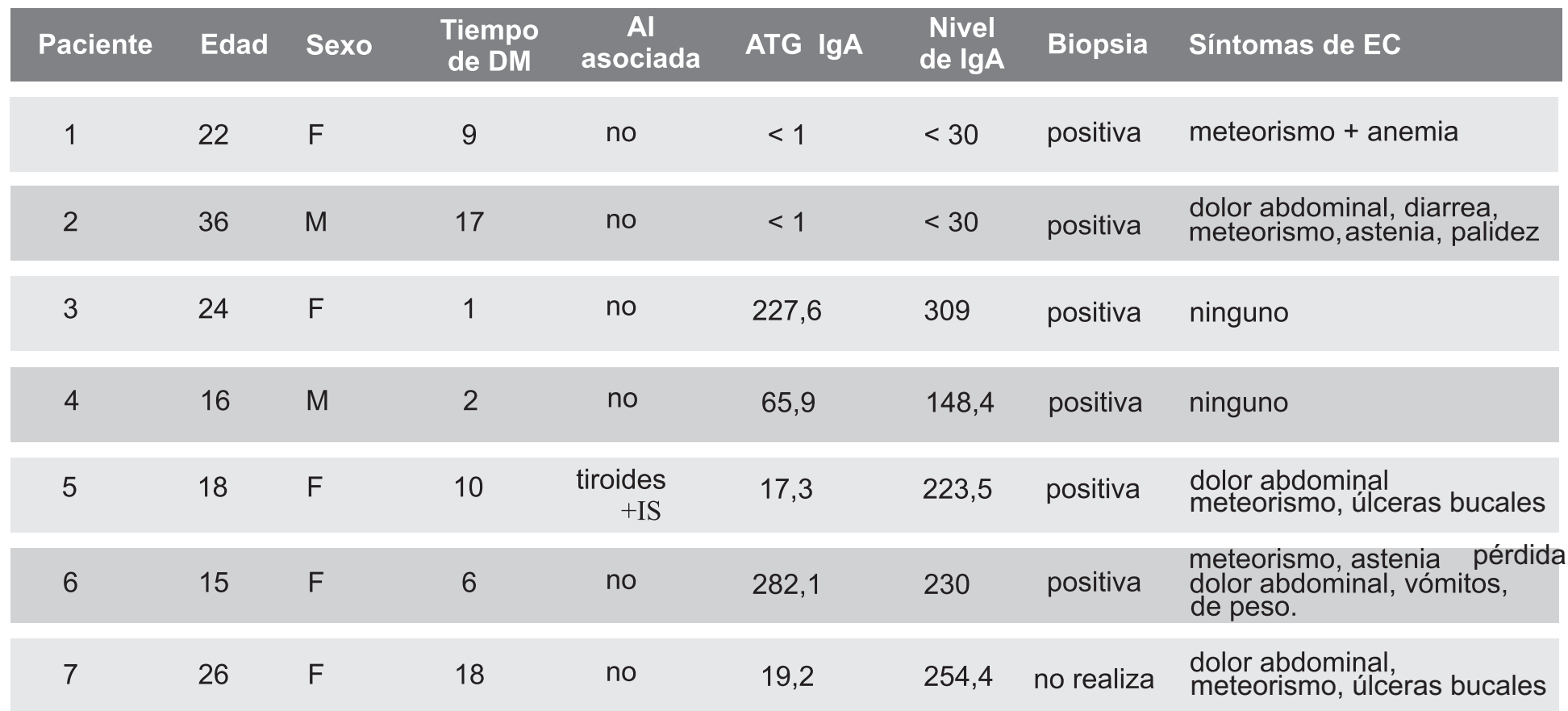

Al: autoinmunidad; IS: insuficiencia suprarrenal; ATG: antic. antitransglutaminasa; tiempo de DM en años; IgA normal: 90-450 $\mathrm{mg} / \mathrm{dl}$.

Las manifestaciones clínicas de EC en DM1 con biopsia positiva se observan en el gráfico 4. 


\section{GRÁFICO 4}

\section{Presentación clínica de enfermedad celiaca (n 6)}

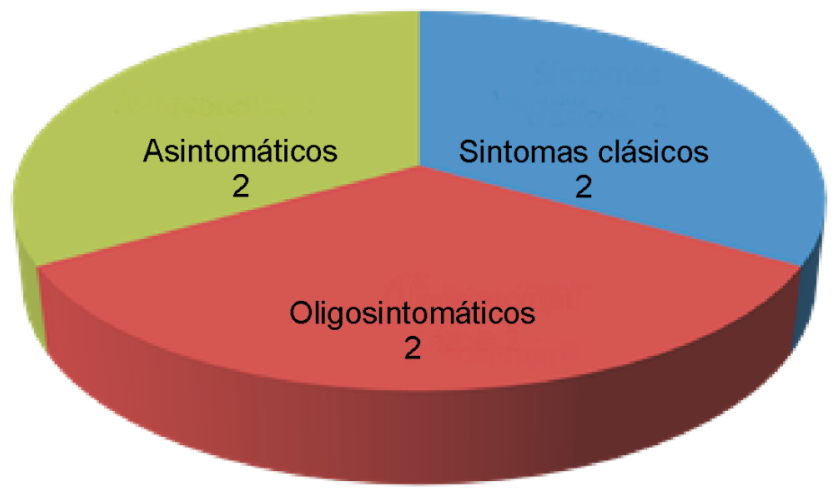

Con respecto al control metabólico en base al promedio de HbA1c y eventos de hipoglucemia, se pueden observar los resultados comparados de ambos grupos en la tabla 3. No se encontraron diferencias significativas en el control metabólico (HbA1c) ni en eventos de hipoglucemia.

\section{TABLA 3}

\section{Comparación entre diabetes mellitus tipo 1 y enfermedad celiaca}

\begin{tabular}{lccc}
\hline \multicolumn{1}{c}{ Características } & Con DM1 y EC (n o) & Con DM1 y sin EC (n 9) Valor p \\
\hline Edad (años) & $21,8 \pm 7,7(15$ a 36$)$ & $26,5 \pm 8,5(17$ a 40$)$ & NS \\
Sexo F/M & $4(67 \%) / 2(33 \%)$ & $5(56 \%) / 4(44 \%)$ & NS \\
$\begin{array}{l}\text { Duración de DM1 } \\
\text { (meses) }\end{array}$ & $90 \pm 70,6(12$ a 204$)$ & $109,3 \pm 62,4(24$ a 192$)$ & NS \\
Autoinmunidad asociada & $1(16,6 \%)$ & $1(11,1 \%)$ & NS \\
Deficiencia de IgA & $2(33,3 \%)$ & $0(0 \%)$ & \\
Nivel de IgA & $161,8 \pm 114,1(30$ a 309$)$ & $270 \pm 102,5(162$ a 490$)$ & NS \\
Talla & $1,59 \pm 0,07(1,52$ a 1,7$)$ & $1,66 \pm 0,09(1,56$ a 1,81$)$ & NS \\
IMC & $22,9 \pm 2,7(18,7$ a 26,5$)$ & $23,06 \pm 2,4(19,3$ a 28,05$)$ & NS \\
Síntomas clásicos & $2(33 \%)$ & $0(0 \%)$ & NS \\
Oligo-asintomáticos & $4(66,6 \%)$ & $0(0 \%)$ & NS \\
HbA1C (\%) & $10,01 \pm 2,2(6,9$ a 12$)$ & $9,9 \pm 2,2(6,7$ a 14,5$)$ & NS \\
Eventos de hipoglucemia & $4(66,7 \%)$ & $7(77,8 \%)$ & NS \\
\hline
\end{tabular}

\section{Discusión}

La frecuencia de EC en pacientes con DM1 en nuestro estudio es un porcentaje alto (9,4\%), en comparación con otros estudios que varía entre 1 a $8 \% .^{4-9}$ Un estudio realizado en el Brasil encontró una prevalencia del $2,5 \%{ }^{4}$, mientras que otro en España halló una prevalencia del $8 \%{ }^{5}$, existiendo series que muestran prevalencia de hasta $12 \%{ }^{7}$. Todas estas investigaciones fueron realizadas en niños y adolescentes diabéticos, a diferencia de nuestra población que estuvo enfocada en adolescentes y adultos, con una media de edad de 25 años y un promedio de 8,5 años de diabetes, lo que pudiera explicar esta mayor tasa de asociación. 
De acuerdo al estudio multicéntrico de Cerutti y cols. ${ }^{6}$, la EC fue encontrada en el 75\% de los niños dentro de los 4 años del diagnóstico de DM1 y raramente encontrado luego de 10 años de duración. El tiempo de aparición de la EC, siguiendo al desarrollo de la DM1, es impredecible. Se debería seguir con el tamizaje regular, incluso cuando los niños llegan a la adolescencia y a la edad adulta.,

De los 6 pacientes con EC, 2 presentaban los “clásicos síntomas" de esta enfermedad, mientras que entre los demás, 2 eran totalmente asintomáticos y 2 oligosintomáticos, con síntomas inespecíficos y aislados. Estos resultados coinciden con otros estudios similares, demostrando que la mayoría de los pacientes diabéticos con enfermedad celiaca refieren pocos o ningún síntoma de mala absorción, y cuando están presentes son leves y rescatados retrospectivamente. ${ }^{2,5,8,11}$ Teniendo en cuenta que los síntomas clínicos no tienen correlación con la biopsia positiva, el diagnóstico de EC en pacientes con DM1 no puede estar basado en la aparición de síntomas solamente, debe ser buscado por medio del dosaje de anticuerpos y confirmado por la endoscopía con toma de biopsia duodenal. El tamizaje puede detectar formas atípicas, silentes o asintomáticas de la EC, y esto puede ser beneficioso al reducir el riesgo de potenciales complicaciones, como linfomas y osteoporosis, con el tratamiento precoz con la dieta sin gluten. ${ }^{21-24}$

El control metabólico de nuestros pacientes con DM1 y EC, medido por la HbA1c, no difiere significativamente con el grupo control (10,01 vs 9,9\%). Sigue siendo controversial el efecto de la EC sobre el control glucémico. Se postula que la EC produce un peor control glucemico ${ }^{1}$, pero esto no ha sido demostrado por otros estudios, que en la mayoría de los casos no encuentra diferencias entre diabéticos con y sin EC. ${ }^{8,11,12}$ Una posible explicación es que en aquellos con DM1 y EC hay una mayor tendencia al mal control glucémico (alta HbA1c) y son más propensos a presentar episodios de hipoglucemia (baja HbA1c) según el grado de mala absorción de nutrientes, pudiendo esto resultar en una HbA1c menor a lo esperado. ${ }^{8}$ En nuestro estudio tampoco encontramos diferencia significativa en el control metabólico, pero la pequeña cantidad de pacientes no nos permite llegar a conclusiones definitivas.

Otro punto de comparación en nuestro estudio fueron los eventos de hipoglucemia, no encontrando diferencia significativa con el grupo control, incluso la frecuencia de eventos fue mayor en el grupo sin EC pero sin ser significativa. El riesgo de hipoglucemia severa es mayor en los pacientes diabéticos con EC sintomática, debido a la absorción errática de nutrientes, ${ }^{2,3,10}$ sin embargo, el efecto de la EC subclínica en diabéticos con respecto a la frecuencia de eventos de hipoglucemia sigue siendo poco clara. Iafusco y cols. ${ }^{14}$ encontraron mayor número de hipoglucemias en el periodo comprendido entre los 6 meses previos al diagnostico de EC y los 6 meses después de iniciada la dieta libre de gluten, y posteriormente fueron retornando a niveles comparables. Otros estudios también encontraron más eventos de hipoglucemia en pacientes con EC. ${ }^{11,22}$ Teniendo en cuenta los resultados de estos estudios, parecería ser que los eventos de hipoglucemia son más frecuentes en DM1 con EC.

Encontramos una alta frecuencia de EC en nuestra población con DM1, la mayoría sin síntomas característicos. La presencia de EC parece no repercutir en el control metabólico de la diabetes ni en la frecuencia de eventos de hipoglucemia, pero la pequeña cantidad de pacientes con DM1 y EC es un factor limitante en nuestro estudio, no permitiendo llegar a conclusiones definitivas. A pesar de ello, teniendo en cuenta los resultados de otros estudios y ante la posibilidad de aparición de complicaciones a largo plazo, como la osteoporosis y los linfomas, debemos realizar el cribado sistemático en todos los pacientes diagnosticados de DM1, incluso en la edad adulta. ${ }^{21}$

Nuestros resultados apoyan la práctica regular del tamizaje para EC en todos los pacientes con DM1, dada la alta frecuencia de asociación. No debemos basarnos en lo síntomas clásicos, ya que la mayoría son oligoasintomáticos. No encontramos efecto de la EC sobre el control metabólico y los eventos de hipoglucemia. 


\section{Nota}

Este artículo obtuvo el Premio Carlos Esteban Arbo Sosa al mejor tema libre del XI Congreso Paraguayo de Medicina Interna (Asunción, Paraguay, abril 2012).

\section{Referencias bibliográficas}

1. Schwarzenberg SJ, Brunzell C. Type 1 Diabetes and Celiac Disease: Overview and Medical Nutrition Therapy. Diabetes Spectrum 2002; 15(3):197-201

2. Freemark M, Levitsky LL. Screening for celiac disease in children with type 1 diabetes: two views of the controversy. Diabetes Care. 2003 Jun; 26(6): 1932-9.

3. Collin P, Kaukinen K, Välimäki M, Salmi J. Endocrinological disorders and celiac disease. Endocr Rev. 2002 Aug; 23(4): 464-83.

4. Mont-Serrat C, Hoineff C, Meirelles RM, Kupfer R. Diabetes and autoimmune diseases: prevalence of celiac disease in children and adolescents with type 1 diabetes. Arq Bras Endocrinol Metabol. 2008 Dec; 52(9): 1461-5.

5. Nóvoa Medina Y, López-Capapé M, Lara Orejas E, Alonso Blanco M, Camarero Salces C, Barrio Castellanos R. Impact of diagnosis of celiac disease on metabolic control of type 1 diabetes. An Pediatr (Barc). 2008 Jan; 68(1): 13-7.

6. Cerutti F, Bruno G, Chiarelli F, Lorini R, Meschi F, Sacchetti C; Diabetes Study Group of the Italian Society of Pediatric Endocrinology and Diabetology. Younger age at onset and sex predict celiac disease in children and adolescents with type 1 diabetes: an Italian multicenter study. Diabetes Care. 2004 Jun; 27(6): 1294-8.

7. Hansen D, Brock-Jacobsen B, Lund E, Bjørn C, Hansen LP, Nielsen C, Fenger C, Lillevang ST, Husby S. Clinical benefit of a gluten-free diet in type 1 diabetic children with screening-detected celiac disease: a population-based screening study with 2 years' follow-up. Diabetes Care. 2006 Nov; 29(11): 2452-6.

8. Goh C, Banerjee K. Prevalence of coeliac disease in children and adolescents with type 1 diabetes mellitus in a clinic based population. Postgrad Med J. 2007 Feb; 83(976): 132-6.

9. Cronin CC, Shanahan F. Insulin-dependent diabetes mellitus and coeliac disease. Lancet. 1997 Apr 12; 349(9058): 1096-7.

10. Celiac sprue. Farrell RJ, Kelly CP. N Engl J Med. 2002 Jan 17; 346(3): 180-8.

11. Acerini CL, Ahmed ML, Ross KM, Sullivan PB, Bird G, Dunger DB. Coeliac disease in children and adolescents with IDDM: clinical characteristics and response to gluten-free diet. Diabet Med. 1998 Jan; 15(1): 38-44.

12. Kaukinen K, Salmi J, Lahtela J, Siljamäki-Ojansuu U, Koivisto AM, Oksa H, Collin P. No effect of gluten-free diet on the metabolic control of type 1 diabetes in patients with diabetes and celiac disease. Retrospective and controlled prospective survey. Diabetes Care. 1999 Oct; 22(10): 1747-8.

13. Iafusco D, Rea F, Chiarelli F, Mohn A, Prisco F. Effect of gluten-free diet on the metabolic control of type 1 diabetes in patients with diabetes and celiac disease. Diabetes Care. 2000 May; 23(5): 712-3.

14. Mohn A, Cerruto M, Iafusco D, Prisco F, Tumini S, Stoppoloni O, Chiarelli F. Celiac disease in children and adolescents with type I diabetes: importance of hypoglycemia. J Pediatr Gastroenterol Nutr. 2001 Jan; 32(1): 37 40.

15. Fraser-Reynolds KA, Butzner JD, Stephure DK, Trussell RA, Scott RB. Use of immunoglobulin A-antiendomysial antibody to screen for celiac disease in North American children with type 1 diabetes. Diabetes Care. 1998 Nov; 21(11): 1985-9.

16. Carlsson AK, Axelsson IE, Borulf SK, Bredberg AC, Lindberg BA, Sjöberg KG, Ivarsson SA. Prevalence of IgAantiendomysium and IgA-antigliadin autoantibodies at diagnosis of insulin-dependent diabetes mellitus in Swedish children and adolescents. Pediatrics. 1999 Jun; 103(6 Pt 1):1248-52.

17. Schober E, Bittmann B, Granditsch G, Huber WD, Hüppe A, Jäger A, Oberhuber G, Rami B, Reichel G. Screening by anti-endomysium antibody for celiac disease in diabetic children and adolescents in Austria. J Pediatr Gastroenterol Nutr. 2000 Apr; 30(4): 391-6. 
18. Lampasona V, Bonfanti R, Bazzigaluppi E, Venerando A, Chiumello G, Bosi E, Bonifacio E. Antibodies to tissue transglutaminase C in type I diabetes. Diabetologia. 1999 Oct; 42(10): 1195-8.

19. Seissler J, Schott M, Boms S, Wohlrab U, Ostendorf B, Morgenthaler NG, Scherbaum WA. Autoantibodies to human tissue transgutaminase identify silent coeliac disease in Type I diabetes. Diabetologia. 1999 Dec; 42(12): 1440 -1.

20. Kordonouri O, Dieterich W, Schuppan D, Webert G, Müller C, Sarioglu N, Becker M, Danne T. Autoantibodies to tissue transglutaminase are sensitive serological parameters for detecting silent coeliac disease in patients with Type 1 diabetes mellitus. Diabet Med. 2000 Jun; 17(6): 441-4.

21. Holmes GK. Non-malignant complications of coeliac disease. Acta Paediatr Suppl. 1996 May; 412: 68-75.

22. Iafusco D, Rea F, Prisco F. Hypoglycemia and reduction of the insulin requirement as a sign of celiac disease in children with IDDM. Diabetes Care. 1998 Aug; 21(8): 1379-81.

23. Zacur M. Metodología diagnóstica y manejo de la enfermedad celiaca del niño: nuestra experiencia en el Paraguay (tesis doctoral). Asunción. Facultad de Ciencias Médicas. Universidad Nacional de Asunción.1994

24. Real Delor RE, Arza G, Chamorro ME, Dalles I, Ibarra Douglas A. La enfermedad celiaca en el Paraguay. Rev Nac (Itauguá) 2011 Dic; 3(2): 7-15 